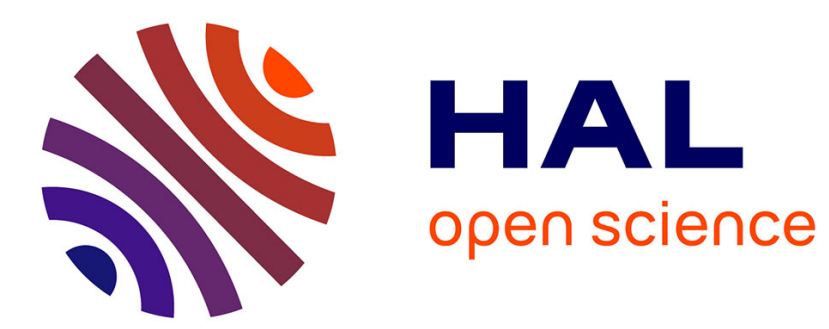

\title{
COMMENTS ON THE ALLOY ANALOGY APPROXIMATION OF THE HUBBARD MODEL
}

\author{
F. Brouers, F. Ducastelle, G. Treglia, M. Brauwers, J. Giner
}

\section{To cite this version:}

F. Brouers, F. Ducastelle, G. Treglia, M. Brauwers, J. Giner. COMMENTS ON THE ALLOY ANALOGY APPROXIMATION OF THE HUBBARD MODEL. Journal de Physique Colloques, 1976, 37 (C4), pp.C4-207-C4-212. 10.1051/jphyscol:1976434 . jpa-00216548

\section{HAL Id: jpa-00216548 https://hal.science/jpa-00216548}

Submitted on 1 Jan 1976

HAL is a multi-disciplinary open access archive for the deposit and dissemination of scientific research documents, whether they are published or not. The documents may come from teaching and research institutions in France or abroad, or from public or private research centers.
L'archive ouverte pluridisciplinaire HAL, est destinée au dépôt et à la diffusion de documents scientifiques de niveau recherche, publiés ou non, émanant des établissements d'enseignement et de recherche français ou étrangers, des laboratoires publics ou privés. 


\title{
COMMENTS ON THE ALLOY ANALOGY APPROXIMATION OF THE HUBBARD MODEL
}

\author{
F. BROUERS
}

Laboratoire de Physique des Solides, 91405 Orsay, France

F. DUCASTELLE and G. TREGLIA

O. N. E. R. A. 92320 Châtillon, France

M. BRAUWERS (*) and J. GINER (**)

Institut de Physique, Université de Liège, Liège 4000 Belgique

\begin{abstract}
Résumé. - Nous prouvons que dans l'approximation d'analogie d'alliage le modèle de Hubbard non dégénéré n'a pas de solution ferromagnétique. Nous montrons qu'il n'y a pas non plus de solution antiferromagnétique. Cependant dans cette approximation la considération d'une interaction d'échange interatomique ou de la dégénérescence orbitale et d'une interaction d'échange intra-atomique peut donner des solutions magnétiques.
\end{abstract}

\begin{abstract}
We prove that within the alloy analogy approximation the non-degenerate Hubbard model has no ferromagnetic solution. We show that contrary to what has been claimed recently there is no CPA antiferromagnetic solution. However in the alloy analogy approximation the consideration of exchange interatomic interaction or the orbital degeneracy and the intraatomic exchange interaction can yield magnetic solutions.
\end{abstract}

1. Introduction. - In this paper we summarize some recent results obtained for the non-degenerate and degenerate Hubbard model using the techniques of the theory of disordered alloys. These results lead to the conclusion that the alloy analogy approximation introduced by Hubbard and used to discuss Mott insulators and the metal-insulator transition does not yield any ferromagnetic or antiferromagnetic solutions for a non-degenerate band

Fukuyama and Ehrenreich [1] have shown that the CPA calculation of the correlations (Hubbard's scattering correction approximation) gives a spin susceptibility which does not exhibit any ferromagnetic instability for any single-band model and carriers number, the case of the $T=0$ half-filled band depending on the limit chosen.

Brouers and Ducastelle [2], have shown that Fukuyama and Ehrenreich's conclusions can be obtained in a staightforward manner by relating the spin susceptibility to the partial Green's functions of the alloy analogy. Using this procedure, one can show that this statement can be more general since we prove that the exact solutions of the alloy analogy approximation of the Hubbard model would yield a susceptibility which would never exhibit any instability, if the Fermi level lies in a region of non-zero density of states. Brouers et al. $[3,4]$ have considered in the same model the

(*) Aspirant F. N. R. S.

$(* *)$ ESIS Fellow. response of the magnetically disordered phase to a periodical external magnetic field. By calculating the wave-vector dependent susceptibility, they have shown that antiferromagnetic ordering is not possible in a half-filled band. This result contradicts Gupta et al. [5] is statement and calculations. We have redone their self-consistent calculations and found no antiferromagnetic solutions.

We have then considered in the same formalism the influence of interatomic interactions and of an intra atomic exchange interaction and degeneracy. One can see in that case that the susceptibility may diverge leading to magnetic solutions when Coulomb and exchange interactions are large enough.

2. Model. - We consider the alloy analogy approximation of the Hubbard model. We assume that the motion of an electron with spin $s= \pm \sigma$ can be described by the following alloy Hamiltonian

$$
\begin{aligned}
\mathcal{H}_{\mathrm{s}}=W_{\mathrm{s}}+\mathcal{H}_{\mathrm{s}}^{\mathrm{e}-\mathrm{e}}= & \sum_{n \neq m} W_{n m} a_{n \mathrm{~s}}^{+} a_{m \mathrm{~s}}+ \\
& +\sum_{n} \varepsilon_{n \mathrm{~s}} a_{n \mathrm{~s}}^{+} a_{n \mathrm{~s}} \quad \mathrm{~s}= \pm \sigma \quad(1,
\end{aligned}
$$

where $\varepsilon_{n \sigma}$ is equal to $\varepsilon^{\mathrm{B}} \equiv u$ or $\varepsilon^{\mathrm{A}} \equiv 0$, depending on whether an electron with spin $-\sigma$ is at site $n$ or not. At a fixed instant in time the electrons with opposite spins are regarded as occupying given positions on randomily distributed lattice sites. One neglects the dynamical effects of opposite spin electrons and one 
replaces the time average which arises because of the continuous rearangement of spins by a configuration average in the alloy problem. Hamiltonian (1) can therefore be viewed as describing two interdependent binary alloys $\mathbf{A}_{1-n-\sigma} \mathbf{B}_{n-\sigma}$. Here $n_{\sigma}$ is the average number of electrons per site with spin $\sigma:\left\langle n_{\sigma}\right\rangle$. The relative concentration of atoms with energy levels $\varepsilon^{\mathrm{A}}$ is $1-n_{-\sigma}$ or $1-n_{\sigma}$ and with energy level $\varepsilon^{\mathrm{B}}$ is $n_{-\sigma}$ or $n_{\sigma}$ corresponding to whether the electrons have spin $\sigma$ or $-\sigma$ respectively. It is assumed for the purpose of calculating the motion of $\sigma$ electrons that electrons of spin $-\sigma$ are fixed at the lattice sites and vice-versa. For a given configuration, one can define a Green's function

$$
G_{ \pm \sigma}=\left(z-\mathscr{H}_{ \pm \sigma}\right)^{-1}
$$

and the average number of electrons with spin $\sigma$ is given by

$$
\begin{aligned}
n_{\sigma}=\left\langle n_{+\sigma}\right\rangle=- & \frac{1}{\pi N} \operatorname{Im} \operatorname{Tr} \times \\
& \times \int_{-\infty}^{E_{F}} \mathrm{~d} E\left\langle G_{\sigma}\left(E, n_{-\sigma}\right)>\right.
\end{aligned}
$$

where $N$ is the number of sites. The average Green's function being a function of the concentration $n_{-\sigma}$ is given by (at $T=0$ )

$$
n_{-\sigma}=-\frac{1}{\pi N} \operatorname{Im} \operatorname{Tr} \int_{-\infty}^{E_{\mathcal{F}}} \mathrm{d} E\left\langle G_{-\sigma}\left(E, n_{\sigma}\right)\right\rangle
$$

the Fermi level is defined by a third equation

$$
n=n_{\sigma}+n_{-\sigma}
$$

where $n$ is the average number of electrons per site.

3. Static spin susceptibility. - The paramagnetic spin susceptibility corresponding to an uniform magnetic field $H$ is written as

$$
\chi=\left.\mu_{\mathrm{B}} \frac{n_{\sigma}-n_{-\sigma}}{H}\right|_{H \rightarrow 0}=2 \mu_{\mathrm{B}} \frac{\mathrm{d} n_{\sigma}}{\mathrm{d} H}
$$

and $n_{\sigma}$ is given in terms of $\left\langle G_{\sigma}\right\rangle$ and the Fermi energy by

$$
\begin{aligned}
n_{\sigma}(H)=- & \frac{1}{\pi N} \operatorname{Im} \operatorname{Tr} \times \\
& \times \int_{-\infty}^{E_{F}} \mathrm{~d} E<G_{\sigma}\left(E+\mu_{B} \sigma H, n_{-\sigma}\right)>.
\end{aligned}
$$

We therefore have

$$
\frac{\mathrm{d} n_{\sigma}}{\mathrm{d} H}=\frac{\partial n_{\sigma}}{\partial H}+\frac{\partial n_{\sigma}}{\partial n_{-\sigma}} \cdot \frac{\mathrm{d} n_{-\sigma}}{\mathrm{d} H}
$$

and

$$
\chi=\frac{2 \mu_{\mathrm{B}} \frac{\partial n_{\sigma}}{\partial H}}{1+\frac{\partial n_{\sigma}}{\partial n_{-\sigma}}}=2 \mu_{\mathrm{B}}^{2} g_{\sigma}\left(E_{\mathrm{F}}\right)\left[1+\frac{\partial n_{\sigma}}{\partial n_{-\sigma}}\right]^{-1}
$$

Since from eq. (7), one finds

$$
\frac{\partial n_{\sigma}}{\partial H}=\mu_{\mathrm{B}} g_{\sigma}\left(E_{\mathrm{F}}\right)
$$

where $\mu_{\mathrm{B}}$ is the Bohr magneton and $g_{\sigma}\left(E_{\mathrm{F}}\right)$ the density of states at the Fermi level of $\sigma$ spin electrons

$$
g_{\sigma}\left(E_{\mathrm{F}}\right)=-\frac{1}{\pi N} \operatorname{Im} \operatorname{Tr}\left\langle G_{\sigma}\left(E_{\mathrm{F}}+i o\right)>.\right.
$$

The susceptibility diverges if $\partial n_{\sigma} / \partial n_{-\sigma}$ is equal to -1 . One can show (Brouers and Ducastelle [2]) that this condition is never fulfilled if the density of states at Fermi level is non-zero. If $\left\langle G_{\sigma}(E)\right\rangle$ is the exact alloy average Green's function, one can write

$$
\left.\left\langle\operatorname{Tr} \frac{\mathrm{d} G}{\mathrm{~d} E} G^{-1}\right\rangle=-\langle\operatorname{Tr} G\rangle=\frac{\mathrm{d}}{\mathrm{d} E}<\operatorname{Tr} \log G\right\rangle
$$

and therefore substituting in (3):

$$
n_{+\sigma}=\frac{1}{\pi N} \operatorname{Im} \operatorname{Tr}<\log G_{\sigma}\left(E_{\mathrm{F}}\right)>.
$$

In order to obtain the spin susceptibility we need the derivative of $n_{\sigma}$ with respect to concentration $n_{-\sigma}$.

Quite generally and supposing that the average could be calculated exactly, the derivative of any operator $\hat{o}$ of an alloy $A_{1-c} B_{c}$ with respect to $B$ concentration $c$ is given by

$$
\frac{\mathrm{d}<\hat{o}\rangle}{\mathrm{d} c}=\sum_{n}<\hat{o}_{n=\mathrm{B}}>-<\hat{o}_{n=\mathrm{A}}>
$$

where $\left\langle\hat{o_{n}=\mathrm{B}(\mathrm{A})}\right\rangle$ is the conditional average where site $n$ is occupied by an atom $\mathrm{B}(\mathrm{A})$. One has therefore

$$
\frac{\mathrm{d} n}{\mathrm{~d} c}=-\frac{1}{\pi} \operatorname{Im} \frac{1}{\pi} \sum_{n} \operatorname{Tr}\left\langle\log \frac{G_{n=\mathbf{B}}}{G_{n=\mathrm{A}}}\right\rangle
$$

the two partial Green's functions are related by

$G_{n=\mathrm{B}}=G_{n=\mathrm{A}}+G_{n=\mathrm{A}}\left(\varepsilon_{\mathrm{B}}-\varepsilon_{\mathrm{A}}\right)|n\rangle<n \mid G_{n=\mathrm{B}}$

since for the Hubbard model, $\varepsilon_{\mathrm{A}}=0, \varepsilon_{\mathrm{B}}=U$ and $c=n_{-\sigma}$, it is easy to obtain

$$
\frac{\mathrm{d} n_{\sigma}}{\mathrm{d} n_{-\sigma}}=\frac{1}{\pi} \operatorname{Im}<\log \left(1+U<n\left|G_{n=-\sigma(o c c)}^{\sigma}\right| n>\right)>
$$

where $G_{n=-\sigma(\text { occ })}$ is the partial Green's function of a $\sigma$ electron on site $n$ occupied by an electron of spin $-\sigma$. As the imaginary part of this quantity is always negative inside the band, it is straight-forward to show that if the density of states is non zero, one has always

$$
-1<\frac{\mathrm{d} n_{\sigma}}{\mathrm{d} n_{-\sigma}}<0
$$

and therefore that the susceptibility is never divergent inside the band. The statement is more general than the one given by Fukuyama and Ehrenreich since we did not use any specific approximation for the average 
Green's function. One can show that CPA yields a final expression for $\mathrm{d} n_{\sigma} / \mathrm{d} n_{-\sigma}$

$$
\frac{\mathrm{d} n_{\sigma}}{\mathrm{d} n_{-\sigma}}=\frac{1}{\pi} \operatorname{Im} \log \left(1+U F_{n=-\sigma(o c c)}^{\sigma}\right)
$$

which is similar to (17) if the average is the alloy CPA average and $F$ the average diagonal Green's function. The conclusions are obviously identical.

For a precisely half-filled band $\chi$ is not determined uniquely. If we consider the limit $n \rightarrow 0.5$, it can be shown that the susceptibility remains finite.

If there is no ferromagnetism at $T=\mathbf{0}$, obviously the susceptibility does not diverge at $T \neq 0$. This can be proved easily. We start from the definition of the thermodynamical potential

$$
\Omega=-\int f(E) n(E) \mathrm{d} E=U-T S-\mu n
$$

with

$n(E)=\int^{E} g\left(E^{\prime}\right) \mathrm{d} E^{\prime}$ and $f(E)=\left[1+\mathrm{e}^{(E-\mu) / k T}\right]^{-1}$

is the chemical potential and $n$ is the number of electrons.

$$
\text { At } T=0
$$

$$
\Omega(T=0)=U-\mu n
$$

and the number of electrons is given by

$$
n=n(\mu)=-\frac{\partial \boldsymbol{\Omega}}{\partial \mu} \text {. }
$$

At $T \neq 0$

$$
n=\int_{-\infty}^{\infty} \frac{\mathrm{d} f(E)}{\mathrm{d} E_{\mathrm{F}}} n(E) \mathrm{d} E
$$

identifying $\mu$ with the Fermi energy and

$$
\frac{\partial n}{\partial c}=\int_{-\infty}^{\infty} \frac{\mathrm{d} f(E)}{\mathrm{d} E_{\mathrm{F}}} \frac{\partial n(E)}{\partial c} \mathrm{~d} E .
$$

In the alloy analogy of the Hubbard model from (25) we can write

$$
\frac{\partial n_{\sigma}}{\partial n_{-\sigma}}=\int_{-\infty}^{\infty} \frac{\mathrm{d} f(E)}{\mathrm{d} E_{\mathrm{F}}} \frac{\partial n_{\sigma}(E)}{\partial n_{-\sigma}} \mathrm{d} E
$$

we have shown at $T=0$ that

$$
-1<\frac{\partial n_{\sigma}(E)}{\partial n_{-\sigma}}<0
$$

for any energy $E$ corresponding to a non zero density of states. Since

$$
\int_{-\infty}^{\infty} \frac{\mathrm{d} f(E)}{\mathrm{d} E_{\mathrm{F}}} \mathrm{d} E=1,
$$

one has therefore for any $T$

$$
-1<\frac{\partial n_{\sigma}}{\partial n_{-\sigma}}<0
$$

4. q-Dependent spin susceptibility (Brouers et al. [4]). - We now consider the q-dependent susceptibility. A periodic external field induces in the paramagnetic phase periodic variation in the number of up and down spin electrons. In the alloy analogy this corresponds to concentration variations. If we start from the disordered paramagnetic phase, we can calculate the spin susceptibility corresponding to periodic spin dependent concentration $n_{-\sigma}$ in the alloy analogy approximation

$$
n_{-\sigma}(\mathbf{q})=c(\mathbf{q})=\frac{1}{N} \sum_{n} \mathrm{e}^{-i \mathbf{q} \mathbf{R}_{\mathbf{n}}} c_{n}
$$

induced by an infinitésimal external field

$$
\mathrm{d} h(\mathbf{q})=\frac{1}{N} \sum_{n} \mathrm{~d} h_{n} \mathrm{e}^{-i \mathbf{q} \mathbf{R}_{n}} .
$$

The q-dependent spin susceptibility reads

$$
\chi(\mathbf{q})=\frac{2 \mu_{\mathbf{B}}^{2} \frac{\partial n_{\sigma}(\mathbf{q})}{\partial h(\mathbf{q})}}{1+\frac{\partial n_{\sigma}(\mathbf{q})}{\partial n_{-\sigma}(\mathbf{q})}} .
$$

In the alloy analogy model $n_{-\sigma}(\mathbf{q})$ plays the role of a concentration. One has

$$
K(\mathbf{q})=\frac{\partial n_{\sigma}(\mathbf{q})}{\partial n_{-\sigma}(\mathbf{q})}=-\frac{1}{\pi} \operatorname{Im} \int_{-\infty}^{E_{\mathrm{F}}} \frac{\delta G_{\sigma}(\mathbf{q}, E)}{\delta c(\mathbf{q})} \mathrm{d} E
$$

$\delta G_{\sigma}(\mathbf{q})$ is the Fourier transform of $\delta\langle n|\bar{G}| n\rangle$ where

$$
\bar{G}=(z-W-\Sigma)^{-1}
$$

in the CPA. The self-energy is site diagonal and one has

$$
\left.\delta<n|\bar{G}| n\rangle=\sum_{m}(<n|\bar{G}| m\rangle\right)^{2} \delta \sigma_{m}
$$

with $\Sigma\left|n>=\sigma_{n}\right| n>$.

Defining the Fourier transform

$$
\mathcal{A}(\mathbf{q})=\sum_{\mathbf{R}} \bar{G}^{2}(R) \mathrm{e}^{-i \mathbf{q} \mathbf{R}} .
$$

The q-dependent spin enhanced susceptibility is defined by

$$
\chi(\mathbf{q})=\frac{\chi^{o}(\mathbf{q})}{1+K(\mathbf{q})}
$$

where $\chi^{0}(\boldsymbol{q})$ is the non-interacting susceptibility and

$$
K(\mathbf{q})=-\frac{1}{\pi} \operatorname{Im} \int_{-\infty}^{E_{F}} \mathcal{A}(\mathbf{q}, E) \frac{\delta \sigma(\mathbf{q}, E)}{\delta c(\mathbf{q})} \mathrm{d} E
$$

where

$$
\sigma(\mathbf{q})=\frac{1}{N} \sum_{n} \mathrm{e}^{-i \mathbf{q} \cdot \mathbf{R}_{n}} \sigma_{n} .
$$

This allows a calculation of a possible instability of the paramagnetic susceptibility for any $\mathbf{q}$. 
To calculate $K(q)$, we expand the self-consistent equation as a function of the concentration fluctuations $\delta c_{n}=c_{n}-c_{n}^{(0)}$ in the completely disordered paramagnetic state. To achieve that programme, we can use an extension of the CPA to inhomogeneous systems discussed in ref. $[3,4,5]$.

One obtains for $\delta c(q)$ the expression in terms of complete disordered quantities ${ }^{(0)}$

$$
\delta \sigma(\mathbf{q})=\frac{\delta c(\mathbf{q}) \Delta t^{(0)}}{1-<t^{2}>^{(0)} \mathcal{A}^{\prime}(\mathbf{q})}
$$

where

$$
\Delta t^{(0)}=t_{\mathrm{B}}^{(0)}-t_{\mathrm{A}}^{(0)}\left[t_{i}^{(0)}=\left(\varepsilon_{i-\sigma}\right) /\left(1-\left(\varepsilon_{i-\sigma}\right) \bar{G}_{n n}^{(0)}\right)\right]
$$

is the difference of $t$-matrices on sites occupied by $\mathrm{A}$ or $B$ atoms and

$$
\mathfrak{A}^{\prime}(\mathbf{q})=\sum_{n \neq m}\left(<n\left|\bar{G}^{(0)}\right| m>\right)^{2} \mathrm{e}^{i \mathbf{q}\left(\mathbf{R}_{n}-\mathbf{R}_{m}\right)} .
$$

This gives finally

$$
\begin{aligned}
K(\mathbf{q})=-\frac{1}{\pi} \operatorname{Im} & \int_{-\infty}^{E_{F}} \mathcal{A}(\mathbf{q}, E) \times \\
& \times \frac{\Delta t^{(0)}}{1-<t^{2}>^{(0)} \mathcal{A}^{\prime}(\mathbf{q}, E)} \mathrm{d} E .
\end{aligned}
$$

We have calculated this quantity in a one-electron band model with nearest neighbour interactions on a $\mathrm{BCC} \mathrm{ClCs} \mathrm{structure} \mathrm{for} \mathrm{a} \mathrm{wave-vector} \mathrm{corresponding} \mathrm{to}$ antiferromagnetic order $\mathbf{q}^{*}=2 \pi / a \quad(1,1,1)$ (see ref. $[3,4])$.

The functions $K\left(\mathbf{q}^{*}\right)$ and $\chi\left(\mathbf{q}^{*}\right)$ have been calculated for that model using a semi-elliptic density of states for a half-filled band. The function in terms of $U$ is represented by figure 1 . It diverges at $U=0$ due to the presence of a gap in the BCC one-band and decreases slowly

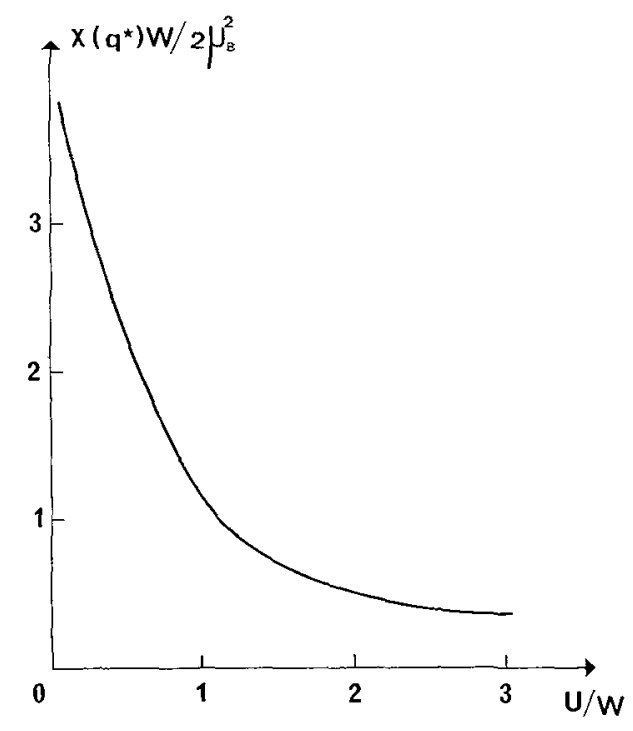

FIG. 1 with $U$. The asymptotic behaviour of $\mathrm{K}\left(\mathbf{q}^{*}\right)$ and $\chi\left(\mathbf{q}^{*}\right)$ can be calculated analytically (cf. ref. [4]) and one gets

$$
\begin{gathered}
\chi\left(\mathbf{q}^{*}\right) \rightarrow \frac{1}{U} 2 \mu_{\mathrm{B}}^{2} \\
U \rightarrow \infty .
\end{gathered}
$$

This result contradicts a recent calculation of Gupta et al. [6] who claim that an antiferromagnetic solution is possible in the non-degenerate Hubbard model within the alloy analogy approximation when $U$ is larger than some critical value. We have redone their calculations and we do not find any antiferromagnetic solution. We discuss that point in the next section.

5. Looking for a CPA self-consistent antiferromagnetic solution. - It is possible to extend the alloy analogy to investigate the possibility of antiferromagnetic solutions. The lattice is divided into two sublattices $\alpha$ and $\beta$. For a half-filled band if one considers the motion of a particular spin, the opposite spin electrons being regarded as frozen, the probabilities of opposite spin occupations are related to the sublattice magnetization and measured by the deviations of the probabilities from the completely disordered ones. One has:

$$
\begin{aligned}
& <n_{\alpha \sigma}>=\left\langle n_{\beta-\sigma}\right\rangle=\frac{1}{2}(1+m) \\
& \left.<n_{\alpha-\sigma}\right\rangle=\left\langle n_{\beta \sigma}\right\rangle=\frac{1}{2}(1-m) .
\end{aligned}
$$

The order parameter has to be calculated selfconsistently.

One may use the CPA theory of Brouers et al. [7]. This was done by Gupta et al. [6]. The disorder self consistency is expressed as usual by the CPA $t$-matrix conditions on both sublattices

$$
\left\langle t_{\alpha}\right\rangle=\left\langle t_{\beta}\right\rangle=0
$$

defining two self-energies $\Sigma_{\alpha}, \Sigma_{\beta}$.

For each spin one can define site-diagonal partia Green'functions corresponding to the two sublattices $F_{\alpha \alpha}\left(\Sigma_{\alpha}, \Sigma_{\beta}\right)$ and $F_{\beta \beta}\left(\Sigma_{\alpha}, \Sigma_{\beta}\right)$ the imaginary part of which define the partial densities of states $g_{\alpha}(E)$ and $g_{\beta}(E)$.

For symmetry reasons the antiferromagnetic selfconsistent condition reads then

$$
m=n_{\alpha}-n_{\beta}=\int_{-\infty}^{E_{F}}\left[g_{\alpha}(E)-g_{\beta}(E)\right] \mathrm{d} E
$$

Since there is a contradiction between the conclusions of the susceptibility calculations reported in section 4 and Gupta et al. [6] results, we have redone their selfconsistent calculations for the same density of states model and which has been characterized in section $4 \mathrm{We}$ find no antiferromagnetic solutions (*). Figure 2 illustrates the results. By contrast the Hartree-Fock approximations yields a antiferromagnetic solution. On this point as well as for the density of state calculations we agree with the numerical results of Gupta $e t$ al. 


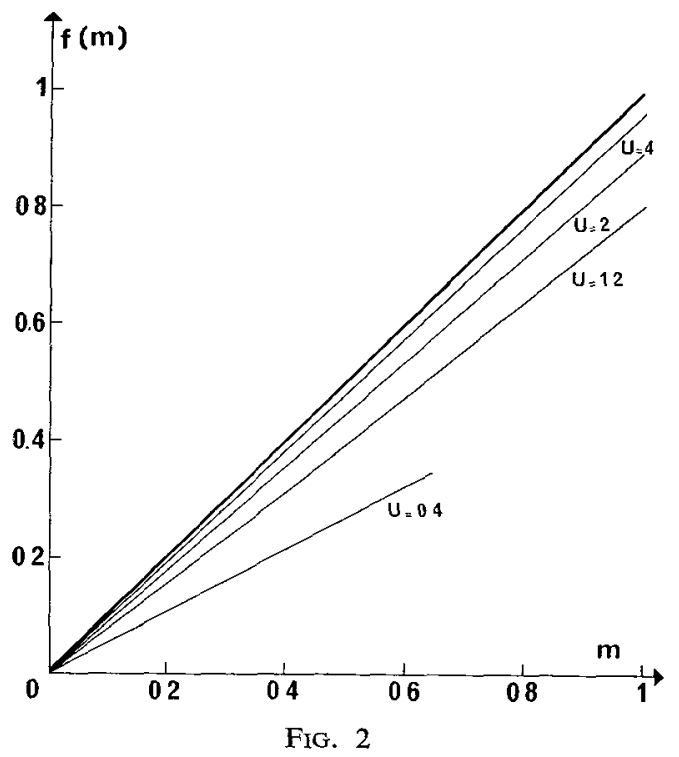

6. Extended Hubbard model. - The Hubbard model with intraatomic exchange interaction can be written

$$
\begin{aligned}
\mathcal{H}=\sum_{\substack{n, m \\
\sigma}} W_{n m}^{\sigma} a_{n \sigma}^{+} a_{m \sigma}+U \sum_{n, \sigma} n_{n \sigma} n_{n-\sigma}+ \\
+U^{(1)} \sum_{\substack{n, m \\
\sigma}} n_{n \sigma} n_{m-\sigma}+U^{(2)} \sum_{\substack{n, m \\
\sigma}} n_{n \sigma} n_{m, \sigma} .
\end{aligned}
$$

Since the two interatomic interactions are much smaller than the intraatomic interaction $U$ one can use the Hartree-Fock approximation to investigate the influence of $U^{(1)}$ and $U^{(2)}$ on the CPA susceptibilities. In that case defining

$$
\widetilde{U}=U+Z U^{(1)} \text { and } \widetilde{U}^{(2)}=-Z U^{(2)},
$$

the two levels corresponding to an electron with spin $\downarrow$ are $-\tilde{U}^{(2)}<n_{\sigma}>$ with probability $1-<n_{\sigma}>$ and $\left.\widetilde{U}-\widetilde{U}^{(2)}<n_{\sigma}\right\rangle$ with probability $\left\langle n_{-\sigma}\right\rangle$. The susceptibility is now given by

$$
\chi=\frac{2 \mu_{\mathrm{B}}^{2} g\left(E_{\mathrm{F}}\right)}{1+\frac{\partial n_{\sigma}}{\partial n_{-\sigma}}-\widetilde{U}_{2} g\left(E_{\mathrm{F}}\right)} .
$$

As we have seen in section 3 , one has

$$
-1<\frac{\partial n_{\sigma}}{\partial n_{-\sigma}}<0
$$

however the denominator may diverge if $\widetilde{U}^{(2)}$ and the density of states at Fermi level are large enough. Vedyayev et al. [8] have considered the $U \rightarrow \infty$ limit. We have calculated $\chi$ for all values of $U$. For instance if $\widetilde{U}^{(2)} / U=0.1$ and a average electron number $\bar{n}=0.5$, an instability occurs at $U \sim 7$ and the Fermi energy lies in an instability region for $U \sim 12$ (in units of half bandwidth). These are indeed large values. We must notice however that in real systems the density of states can be much larger than the one given by the semielliptic band and that the position of the Fermi level can depend on the electronic population of other bands.

7. Susceptibility for a two-fold degenerate band. Starting from the general expression given by Hubbard [9] and neglecting the spin-flip terms, one can write an alloy analogy approximation of the two orbital Hamiltonian as a set of four effective Hamiltonians (Ref. [2] and [10]) :

$$
\mathcal{H}_{\mathrm{vs}}=W_{v \mathrm{~s}}+\sum_{n} \varepsilon_{n v \mathrm{~s}} n_{n v \mathrm{~s}} \quad v=\alpha \cdot \beta \quad \mathrm{s}= \pm \sigma
$$

with for example

$$
\varepsilon_{\alpha \sigma}=U\left(n_{\alpha-\sigma}+n_{\beta-\sigma}\right)+(U-J) n_{\beta \sigma} .
$$

In that case the spin susceptibility reads

$$
\chi=2 \mu_{\mathrm{B}} \frac{\partial n_{\sigma}}{\partial H}[1+2 a-b]^{-1}
$$

where

$$
a=\frac{\partial n_{\alpha \sigma}}{\partial n_{\alpha-\sigma}}=\frac{\partial n_{\alpha \sigma}}{\partial n_{\beta-\sigma}}
$$

and

$$
b=\frac{\partial n_{\alpha \sigma}}{\partial n_{\beta \sigma}}
$$

Since one can have a maximum of four electrons on each site, the CPA is obtained as the result of a selfconsistency of the diagonal Green's functions over eight possible configurations. To calculate $a$ and $b$ one uses a generalization of (17) to get

$$
\begin{aligned}
& a= \frac{\partial n_{\alpha \sigma}}{\partial n_{\beta-\sigma}}=\frac{1}{\pi} \operatorname{Im} \times \\
& \quad \times<\log \left[1+U<n\left|G_{n=\alpha-\sigma(\mathrm{occ})}^{\alpha \sigma}\right| n>\right]> \\
& b=\frac{\partial n_{\alpha \sigma}}{\partial n_{\beta \sigma}}=\frac{1}{\pi} \operatorname{Im} \times \\
& \quad \times<\log \left[1+(U-J)<n\left|G_{n=\beta \sigma(\text { occ })}^{\alpha \sigma}\right| n>\right]>.
\end{aligned}
$$

By arguments similar to those used in section 3, one can show that

$$
-1<a<0 \text { and }-1<b<0 \text {. }
$$

However the denominater of (51) can become negative yielding an instability of the susceptibility.

The numerical results presented in ref. [2] indicate that if $U$ and $J / U$ are large enough the susceptibility diverges and one can notice the correspondance between the instability of the susceptibility and the tendency of the spin of the two orbitals to be aligned in agreement with Hund's rule.

This formalism can be generalized to a two sublattice system in order to allow for spin and orbital order. The 
virtual crystal approximation yields the Hartree-Fock solution of Cyrot and Lyon-Caen [11] when there is one electron/atom $(\bar{n}=1)$. In ref. [10], one establishes the two-sublattice CPA equations for the degenerate Hubbard model and the Hartree-Fock approximation for any $\bar{n}$. The numerical investigations of the solutions are in progress.

Note added in proof: this can be shown analytically (Ducastelle, to be published).

\section{References}

[1] Fukuyama, H. and Ehrenreich, H., Phys. Rev. B 7 (1973) 3266.

[2] Brouers, F. and Ducastelle, F., J. Physique 36 (1975) 851.

[3] Brouers, F., Ducastelle, F. and Gingr, J., Magnetism Autumn School of the Technical (University of Dresden) 1975.

[3] Brouers, F., Ducastelle, F. and Giner, J., J. Physique (to be published).

[4] Gautier, F., Ducastelle, F. and Giner, J., Phil. Mag. 31 (1975) 1373
[6] Gupta, A. K., Edwards, D. M. and Hewson, A. C., $J$. Phys. C. 8 (1975) 3207.

[7] Brouers, F., Giner, J. and van Der Rest, J. Phys. F 4 (1974) 214.

[8] Vedyayev, A. V., Kondorskit, E. Y. and Mizie, E., Phys. Status Solidi (b) 72 (1975) 205.

[9] Hubbard, J., Proc. Roy. Soc. A 277 (1964) 287.

[10] Brouers, F., Phys. Status Solidi 76 (1976) 145.

[11] Cyrot, M. and Lyon-Cakn, C., J. Physique 36 (1975) 253. 